\title{
A systematic multidisciplinary initiative for reducing the risk of complications in adult scoliosis surgery
}

\author{
Rajiv Sethi, MD, ${ }^{1-3}$ Quinlan D. Buchlak, MPsych, ${ }^{1}$ Vijay Yanamadala, MD, MBA, ${ }^{1}$ \\ Melissa L. Anderson, MS, ${ }^{3}$ Eric A. Baldwin, MS, ${ }^{3}$ Robert S. Mecklenburg, MD, ${ }^{1}$ \\ Jean-Christophe Leveque, MD, ${ }^{1}$ Alicia M. Edwards, MBA, ${ }^{1}$ Mary Shea, MA,${ }^{3}$ Lisa Ross, MPH, ${ }^{3}$ and \\ Karen J. Wernli, $\mathrm{PhD}^{3}$
}

\begin{abstract}
${ }^{1}$ Neuroscience Institute, Virginia Mason Medical Center; ${ }^{2}$ Department of Health Services, University of Washington; and ${ }^{3}$ Group Health Research Institute, Seattle, Washington
\end{abstract}

\begin{abstract}
OBJECTIVE Systematic multidisciplinary approaches to improving quality and safety in complex surgical care have shown promise. Complication rates from complex spine surgery range from $10 \%$ to $90 \%$ for all surgeries, and the overall mortality rate is $1 \%-4 \%$. These rates suggest the need for improved perioperative complex spine surgery processes designed to minimize risk and improve quality.
\end{abstract}

METHODS The Group Health Research Institute and Virginia Mason Medical Center implemented a systematic multidisciplinary protocol, the Seattle Spine Team Protocol, in 2010. This protocol involves the following elements: 1) a comprehensive multidisciplinary conference including clinicians from neurosurgery, anesthesia, orthopedics, internal medicine, behavioral health, and nursing, collaboratively deciding on each patient's suitability for surgery; 2) a mandatory patient education course that reviews the risks of surgery, preparation for the surgery, and postoperative care; 3) a dual-attending-surgeon approach involving 1 neurosurgeon and 1 orthopedic spine surgeon; 4) a dedicated specialist complex spine anesthesia team; and 5) rigorous intraoperative monitoring of a patient's blood loss and coagulopathy. The authors identified 71 patients who underwent complex spine surgery involving fusion of 6 or more levels before implementation of the protocol (surgery between 2008 and 2010) and 69 patients who underwent complex spine surgery after the implementation of the protocol (2010 and 2012). All patient demographic variables, including age, sex, body mass index, smoking status, diagnosis of diabetes and/or osteoporosis, previous surgery, and the nature of the spinal deformity, were comprehensively assessed. Also comprehensively assessed were surgical variables, including operative time, number of levels fused, and length of stay. The authors assessed overall complication rates at 30 days and 1 year and detailed deaths, cardiovascular events, infections, instrumentation failures, and CSF leaks. Chi-square and Wilcoxon rank-sum tests were used to assess differences in patient characteristics for patients with a procedure in the preimplementation period from those in the postimplementation period under a Poisson distribution model.

RESULTS Patients who underwent surgery after implementation of the Seattle Spine Team Protocol had a statistically significant reduction (relative risk 0.49 [95\% $\mathrm{Cl} 0.30-0.78]$ ) in all measured complications, including cardiovascular events, wound infections, other perioperative infections, and implant failures within 30 days after surgery; the analysis was adjusted for age and Charlson comorbidity score. A trend toward fewer deaths in this group was also found.

CONCLUSIONS This type of systematic quality improvement strategy can improve quality and patient safety and might be applicable to other complex surgical disciplines. Implementation of these strategies in the treatment of adult spinal deformity will likely lead to better patient outcomes.

https://thejns.org/doi/abs/10.3171/2016.11.SPINE16537

KEY WORDS risk reduction; health services; surgical complications; spinal surgery; systematic improvement

ABBREVIATIONS ASD = adult spinal deformity; CPT = Current Procedural Terminology; DVT = deep venous thrombosis; EHR = electronic health record; ICD-9-CM = International Classification of Diseases, Ninth Revision, Clinical Modification; PE = pulmonary embolism; RR = relative risk.

SUBMITTED May 9, 2016. ACCEPTED November 7, 2016.

INCLUDE WHEN CITING Published online March 31, 2017; DOI: 10.3171/2016.11.SPINE16537. 
$\mathrm{C}$ OMPLEX spine surgeries in the treatment of adult spinal deformity (ASD) are challenging procedures with high rates of intraoperative and postoperative morbidity, including wound infections and neurological injuries, and death. ${ }^{4,7,8,14,15,18,19,21,23,24}$ The rates of major and minor complications for these procedures range from $10 \%$ to almost $90 \% .^{14,19,21}$ There might be opportunities to decrease such risk in complex spine surgery. Many organizations have developed focused protocols aimed at reducing specific complications individually., ${ }^{1,2}$ A growing body of literature suggests that standardized systematic protocols can reduce complication rates across surgical disciplines. ${ }^{12,13,22,24}$ The implementation of a comprehensive multidisciplinary and systematic approach designed to decrease the full constellation of complications related to spine surgery might contribute to improved care quality and patient outcomes.

We recently published the Seattle Spine Team Protocol, ${ }^{20}$ which is among the first in the field designed to reduce the risk of the most common complications simultaneously. In this study, we rigorously analyzed our data from complex spine surgeries before and after the implementation of this comprehensive multidisciplinary protocol.

American health systems, hospitals, and practitioners are under increasing pressure to move away from fee-forservice-based health care delivery and toward delivering value-driven health care by maximizing quality and safety while holding costs constant. ${ }^{16}$ As requests for transparent high-quality data increase and patients seek institutions that deliver the "best" care, ${ }^{11}$ efforts to improve quality and safety in health care are more important than ever. Innovative multidisciplinary and system-focused approaches represent opportunities for achieving improvements in quality and safety across all fields of medicine.

\section{Methods \\ Study Design and Population}

We conducted a retrospective cohort study of patients who underwent complex spinal surgery between July 1, 2008 , and December 31,2012. The study was a retrospective review of a series of consecutive cases. Complex spinal surgery was defined here as an operation that required either 6 or more levels of vertebral fusion or more than 3 levels of vertebral fusion in a patient with multiple comorbidities. All the patients were members of Group Health Cooperative, an integrated delivery system in the Pacific Northwest. All of the operations were conducted at Virginia Mason Medical Center in Seattle, Washington. The total possible study population included women and men aged 18-85 years with a primary diagnosis of scoliosis (International Classification of Diseases, Ninth Revision, Clinical Modification [ICD-9-CM] 737.1-737.36). We identified 42,609 patients with a diagnosis of scoliosis who were potentially eligible for this study cohort.

\section{Identification of Complex Spine Surgeries}

Among the 42,609 patients with a diagnosis of scoliosis, we identified a subset of them who underwent a complex spine surgery during the study period. Because these procedures are not easily identifiable through bill- ing procedure codes, we used a 2-step approach to capture these cases. First, using automated health record data, we limited the cohort of patients with scoliosis to those who had at least 2 visits to a spinal surgeon 1 year and underwent subsequent multilevel spine fusion surgery, as identified by Current Procedural Terminology (CPT) codes (22532-22819 [spinal fusion], 63710 [graft repair of spine], 22849-2285 [addition of spinal implants], and/or 81.0 [spinal fusion ${ }^{3}$ ]), during our study period. We then used medical record chart abstraction to review the operative reports to determine whether the procedure met our study definition of a complex spine procedure.

For a patient to have undergone spinal surgery, a minimum of 2 neurosurgical visits would have been required, because the preoperative and postoperative visits are separate visits from the actual surgery. Any patients with an absence of 2 neurosurgical visits would not be considered to have undergone spinal surgery and were ineligible for inclusion in our retrospective cohort study on postoperative complications.

There were 666 patients with at least 2 visits to a spine surgeon and subsequent procedure codes for spinal surgery who therefore met our criteria for further chart abstraction review. A trained chart abstractor reviewed all electronic health records (EHRs) of these 666 potential cases. For each surgical case, we abstracted the presence of staged procedures, extent and location of the spinal fusion, the surgeon(s), estimated blood loss, and surgical approach (posterior, anterior, lateral, and combinations). As a result of this review, we excluded 499 patients since their procedure was not considered complex spinal surgery because it involved less extensive fusion procedures, involved fusions to the cervical spine, was for a surgical indication other than scoliosis (e.g., surgery related to recent fracture or cancer), or it was an index procedure conducted at an outside medical facility. If multiple procedures occurred during the study period, the first complex spinal surgery was considered the index spinal surgery. The study cohort therefore comprised 140 patients with scoliosis who had undergone a qualifying complex spine surgery.

\section{Patient Characteristics}

Patient age at the time of surgery and patient sex were available from Group Health Cooperative enrollment and demographic records ${ }^{17}$ Comorbidity and health indicators, including body mass index, current smoking status, and history of myocardial infarction, diabetes, osteoporosis, depression, previous surgical intervention, and/or spondylolisthesis, were assessed from the 2 years before surgery. Charlson/Deyo comorbidity scores were calculated using 1 year of previous health care utilization data at the time of surgery. ${ }^{10}$

\section{Intervention Exposure: Implementation of a Standardized Systematic Protocol}

In 2010, we implemented a standardized systematic protocol at Group Health Cooperative and Virginia Mason Medical Center to mitigate the risk of complications after complex spinal surgery. The key elements of the protocol included the following. ${ }^{20}$ 


\section{Complex Spinal Surgery Conference}

Before surgery, each potential surgical case is presented to a multidisciplinary spine conference with clinicians representing neurosurgery, anesthesia, orthopedics, internal medicine, behavioral health, and nursing. For each case, potential surgical interventions and patient suitability for surgery are discussed among the team. Surgery is delayed for patients not currently suitable for it (e.g., those who require smoking cessation, weight loss, or further medical consultation).

\section{Patient Education Course}

Each patient approved for surgery attends a mandatory education course for patients and their caregivers that reviews preparation for the surgery and postoperative care.

\section{Dual-Surgeon Approach}

For each surgical intervention, 2 attending surgeons with training in neurosurgery and orthopedics perform the procedure concurrently.

\section{Dedicated Specialist Complex Spine Anesthesia Team}

These members of the team play an integral role in the review of each case before, during, and after the multidisciplinary conference and during surgery.

\section{Intraoperative Patient Monitoring}

Patient values for coagulopathy and estimated blood loss are monitored routinely and documented on a whiteboard visible to all operating room members to assess patient stability during the surgery. Intraoperative communication strategies, such as taking an hourly pause for the surgeons and anesthesiologist to review laboratory values and patient status, were also standardized and built into the protocol.

This initiative was designed to maximize patient safety and mitigate the risk of complications, primarily through identifying potential risk factors early, opening clear lines of communication between providers, and standardizing intraoperative protocols to reduce variability. Surgery is delayed for patients not currently suitable for it (e.g., those who require smoking cessation, weight loss, or further medical consultation) and potentially denied for those who are unable to complete medical optimization. Additional detail on the specifics of the protocol were published by Sethi et al. ${ }^{20}$ in 2014 and Buchlak et al. ${ }^{5}$ in 2016.

The goal of this evaluation was to compare patient complication rates in 2008-2010 (the preintervention period) to complication rates in 2011-2012 (the postintervention period) after full implementation of these system improvements in late 2010.

\section{Outcome Measures: Surgical Complications}

Surgical complications were categorized as those that occurred within the first 30 days (1-30 days) or up to 1 year (31-365 days) from the index complex spine surgery. Complications of interest were specified a priori using available literature on surgical complications from complex spinal surgery. ${ }^{14,21}$ Complications were identified through a combination of CPT and ICD-9-CM codes in administrative claims data and EHR review. Complications assessed included postoperative blindness (identified through EHRs only), postoperative CSF leak (ICD-9-CM $339.8,348.4,389.0$, or 792.0), myocardial infarction (ICD9-CM 410), stroke (ICD-9-CM 435), deep venous thrombosis (DVT) (ICD-9-CM 453.40), pulmonary embolism (PE) (ICD-9-CM 415.1), infections, including wound infection (ICD-9-CM 958.3) and pneumonia (ICD-9-CM 486), surgical failure, including implant failure (EHR only) and revision surgery (CPT code 22532, 22819, or 63710), readmission to the hospital (any claim for an inpatient hospital stay in the year after surgery), and death (recorded in the Group Health Research Institute or Washington State death registry).

\section{Statistical Analysis}

The analysis compared complication rates after complex spinal surgery conducted within the preintervention period (2008-2010) to those within the postintervention period (2011-2012) to assess the effects of implementing our systematic spine care protocol intervention. We calculated descriptive statistics of patient characteristics overall and stratified according to study period. We used chi-square tests and Wilcoxon rank-sum tests to assess differences in characteristics between patients who underwent a procedure in the preimplementation period and those whose surgery was within the postimplementation period.

The primary outcome was an overall composite measure defined as the occurrence of any of the prespecified complications and further defined as complications within 30 days and 1 year after the index surgery. If a patient experienced more than 1 complication, only 1 event was counted in the overall composite measure of any complication. Although postoperative blindness is an a priori complication of interest, we had no such complication in our study population and hence did not include blindness as an outcome in the reported results.

Surgical complication rates are presented as the number and proportion of patients with a complication within 30 days and 1 year after surgery. We used a modified Poisson regression model to estimate the relative risk (RR) and $95 \%$ confidence interval (CI) of the primary outcome measure (any complication) to compare surgeries performed in 2011-2012 relative to those performed in 20082010 . The regression model was adjusted for age $(<65 \mathrm{vs} \geq$ 65 ) and Charlson/Deyo comorbidity score (0 vs $\geq 1)$. RRs were estimated only for the composite outcome measure. We did not test for statistical significance between rates of individual complications because they were quite low, and it would not be possible to control for important confounders such as age and comorbidity. Therefore, we chose to conduct statistical testing only for our primary outcome composite measure. All analyses were conducted using Stata 12.0 (StataCorp).

\section{Institutional Review Board Approval}

This study was conducted with approval from our institutional review board and was granted a waiver of written consent. All study procedures were compliant with the Health Insurance Portability and Accountability Act. 


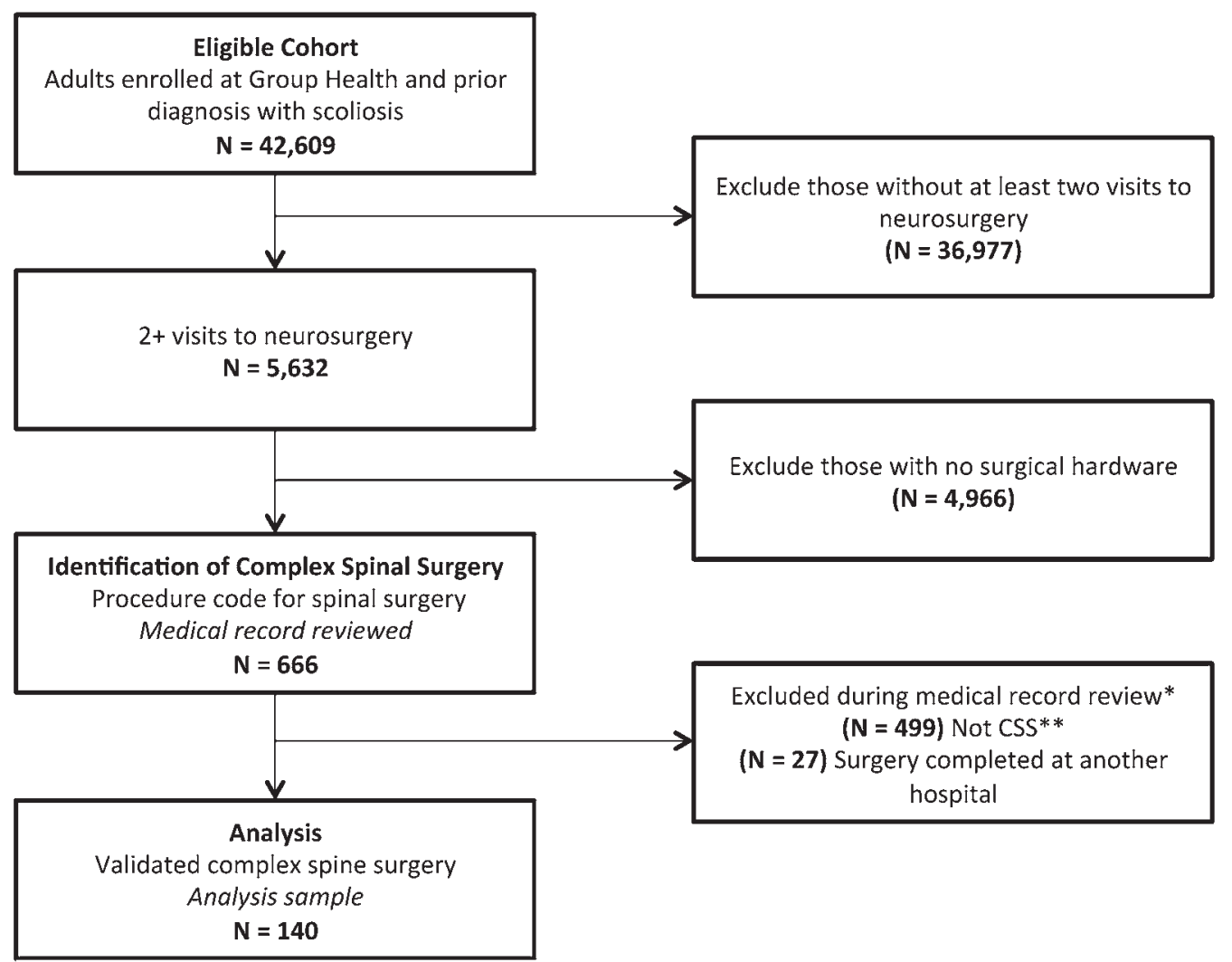

FIG. 1. CONSORT diagram of patients included for evaluation of complex spine surgery. ${ }^{*}$ Patients might have had both exclusions (exclusion identified in the EHR is listed). ${ }^{*}$ Not complex spine surgery (CSS) (1 stage, < 4 levels of fusion; fusion includes cervical spine, scoliosis not indicated, or fracture indicated).

\section{Results}

The study population included 42,609 adult men and women with a diagnosis of scoliosis (Fig. 1). Of those with scoliosis, 5632 patients had 2 visits to a spinal surgeon. Among this cohort, we identified 666 patients who underwent any spine fusion surgery, and after chart review, 140 patients were found to have undergone complex spine surgery and made up our study sample for the analysis.

The study population was $74 \%$ female, accounting for $65 \%$ of the patients in the preintervention period and $84 \%$ in the postintervention period $(\mathrm{p}=0.01)$ (Table 1). Patients who underwent surgery in the postintervention period were more likely to have been a current or recent smoker at the time of their assessment for surgery, more likely to have undergone a previous spinal fusion surgery, and less likely to have been diagnosed with diabetes or previous myocardial infarction, but these differences were not statistically significant.

Surgery characteristics were similar among patients in the preintervention and postintervention periods. On average, the length of hospital stay for the index surgery was 7.6 days. Almost half (46\%) of the surgeries were planned as 2-stage procedures. The number of levels of fusion planned was higher in the postimplementation period; $88 \%$ planned $\geq 6$ levels of fusion compared with $73 \%$ in the preimplementation period $(\mathrm{p}=0.02)$.

The most common complication within 30 days after surgery was CSF leak (Table 2). There were declines in nearly all complications within 30 days after surgery in the postintervention period compared to those in the preintervention period. The most notable reduction was in the 30-day complication rate, primarily because of declines in DVT, PE, wound infection, and return to surgery. There were 4 deaths overall, 3 of which occurred in patients who underwent surgery in the preintervention period.

In the multivariate adjusted model, patients who underwent complex spine surgery in the postintervention period had a statistically significant $51 \%$ decrease in the risk of any complication within 30 days after surgery compared with those who underwent surgery in the preintervention period (RR 0.49 [95\% CI 0.30-0.78]); this analysis was adjusted for age and comorbidity status (Table 3 ). The difference in risk of complications within 1 year between the preintervention and postintervention periods was not statistically significant (RR 0.98 [95\% CI 0.55-1.77]).

\section{Discussion}

The systematic multidisciplinary initiative analyzed here is an evidence-based and scientific solution for improving quality and safety in complex spine surgery care. We conducted a retrospective review of a series of consecutive cases, collected data on several potential confounding factors, accounted for patient differences, and found reductions in overall complication rates within 30 days of surgery. 
TABLE 1. Characteristics of patients who underwent complex spine surgery, overall and according to intervention period

\begin{tabular}{|c|c|c|c|c|}
\hline Characteristic & Overall $(n=140)$ & Preintervention Period $(n=71)$ & Postintervention Period $(n=69)$ & $p$ Value \\
\hline \multicolumn{5}{|l|}{ Patients } \\
\hline Sex (\% female) & 74 & 65 & 84 & 0.01 \\
\hline Age (mean [SD]) (yrs) & $63.7(12.1)$ & $62.0(13.4)$ & $65.5(10.5)$ & 0.09 \\
\hline Body mass index (mean [SD]) $\left(\mathrm{kg} / \mathrm{m}^{2}\right)$ & $27.4(5.5)$ & $28.0(5.6)$ & $26.8(5.3)$ & 0.24 \\
\hline \multicolumn{5}{|l|}{ Personal medical history (\%) } \\
\hline Smoking & 24 & 20 & 29 & 0.20 \\
\hline Diabetes & 12 & 16 & 9 & 0.22 \\
\hline Myocardial infarction & 9 & 11 & 7 & 0.41 \\
\hline Depression & 19 & 18 & 19 & 0.94 \\
\hline Osteoporosis & 15 & 13 & 17 & 0.44 \\
\hline Spondylolisthesis & 59 & 63 & 54 & 0.24 \\
\hline Previous spinal fusion surgery & 19 & 14 & 25 & 0.11 \\
\hline \multicolumn{5}{|l|}{ Charlson/Deyo comorbidity score } \\
\hline 0 & 49 & 45 & 52 & 0.43 \\
\hline $1-2$ & 34 & 39 & 29 & \\
\hline$\geq 3$ & 17 & 15 & 19 & \\
\hline \multicolumn{5}{|l|}{ Surgery } \\
\hline Hospital stay (mean [SD]) (days) & $7.6(4.3)$ & $7.7(4.3)$ & $7.5(4.4)$ & 0.65 \\
\hline \multicolumn{5}{|l|}{ Stages, planned (\%) } \\
\hline 1 & 54 & 55 & 54 & 0.88 \\
\hline 2 & 46 & 45 & 46 & \\
\hline \multicolumn{5}{|l|}{ Levels of fusion, planned (\%) } \\
\hline$<6$ & 20 & 26 & 12 & 0.02 \\
\hline$\geq 6$ & 81 & 73 & 88 & \\
\hline
\end{tabular}

This work contributes to the advancement of health care quality and practice with a specific focus on surgery. The results suggest that our risk mitigation and quality improvement strategies designed to improve patient safety yielded a $51 \%$ reduction in surgical complications within the first 30 days after complex spine surgery. The study revealed decreases in rates of DVT, wound infection, and return to surgery. However, sustained reductions in complication rates were not seen 1 year after surgery, which suggests that the quality improvement measures had an effect on improving patient safety within 30 days of surgery but not within the ensuing year. Our results suggest that quality improvement initiatives can help in the delivery of safer complex spine surgery to patients with scoliosis.

Overall rates of complications at our institution remain lower than benchmarks in the literature. We found 30-day mortality rates of $1 \%$ in the preintervention period and $0 \%$ in the postintervention period and 1-year mortality rates of $3 \%$ in the preintervention period but only $1 \%$ in the postintervention period; previous studies have reported 30 -day mortality rates as high as $4 \% .{ }^{14}$ The review of patients during the preoperative multidisciplinary spine conference helps to determine the suitability of patients for major surgical intervention and enables patients to be optimized for the procedure. Sometimes surgery is deferred until medical health improves. This preoperative review is an important part of the risk management protocol aimed at improving patient safety. ${ }^{20}$

Having a minimum of 2 board-certified neurosurgeons with 10 years of practice experience, a board-certified orthopedic spine surgeon with 10 years of practice experience, 2 board-certified anesthesiologists with specialty training in neuroanesthesia with 10 years of practice experience, 2 board-certified physiatrists, and 1 internist underpins our protocol. We do feel, however, that experience is built with time at each individual institution; thus, rather than stringent experience requirements, only consistent attendance to and involvement with patients with complex spine conditions should be required at centers that desire to build such a multidisciplinary preoperative screening conference. Each center will then need to validate its own outcomes and improve the decision-making process over time.

The primary limitations of this study are that it was retrospective, it was conducted at a single institution, and we could not control for some differences, including sex and levels of surgical fusion planned. In addition, we could not fully account for the increase in surgeon and anesthesiologist experience as the study progressed. We did all that we could to eliminate sampling bias by applying objective patient-selection criteria, which were applicable across the 2 study periods.

We elected to include DVT/PE in a single category to represent "thrombotic" complications. The number of these complications was quite low (10 within the 30-day period and 6 within the 1-year period), so further parsing was unlikely to provide statistical benefit. In addition, to truly represent the clinical scenario, we likely would 
TABLE 2. Complication rates after complex spine surgery 30 days and 1 year after surgery, according to study period

\begin{tabular}{|c|c|c|c|c|}
\hline \multirow[b]{3}{*}{ Complication } & \multicolumn{4}{|c|}{ No. of Patients (\%) } \\
\hline & \multicolumn{2}{|c|}{30 Days } & \multicolumn{2}{|c|}{$1 \mathrm{Yr}$} \\
\hline & Preintervention Period & Postintervention Period & Preintervention Period & Postintervention Period \\
\hline \multicolumn{5}{|l|}{ Immediate } \\
\hline Blindness & $0(0)$ & $0(0)$ & NA & NA \\
\hline CSF leak & $13(18)$ & $12(17)$ & NA & NA \\
\hline \multicolumn{5}{|l|}{ Cardiovascular } \\
\hline Myocardial infarction & $3(4)$ & $0(0)$ & $0(0)$ & $0(0)$ \\
\hline Stroke & $1(1)$ & $1(1)$ & $0(0)$ & $1(1)$ \\
\hline DVT/PE & $7(10)$ & $2(3)$ & $3(4)$ & $3(4)$ \\
\hline \multicolumn{5}{|l|}{ Infection } \\
\hline Wound infection & $6(8)$ & $0(0)$ & $2(3)$ & $1(1)$ \\
\hline Pneumonia & $4(6)$ & $1(2)$ & $2(3)$ & $3(4)$ \\
\hline \multicolumn{5}{|l|}{ Surgical failure } \\
\hline Implant failure & $3(4)$ & $0(0)$ & $3(4)$ & $1(1)$ \\
\hline Return to surgery & $4(6)$ & $1(1)$ & $5(7)$ & $2(3)$ \\
\hline Other & $0(0)$ & $1(1)$ & $0(0)$ & $0(0)$ \\
\hline Readmission to hospital & $7(10)$ & $5(7)$ & $14(20)$ & $12(17)$ \\
\hline Death & $1(1)$ & $0(0)$ & $2(3)$ & $1(1)$ \\
\hline
\end{tabular}

$\mathrm{NA}=$ not applicable.

have needed to separate upper-extremity DVT from lowerextremity DVT and potentially incidental PE discovered on postoperative spine CT from clinically significant PE potentially requiring anticoagulation. We feel that an assessment of thrombotic complications is important but also that the broad categorization used might have more accurately represented the types of complications that our patients face and that can be affected by the use of a systemic protocol.

A key element of this protocol is better patient selection. We have designed and evaluated a protocol that consists of a number of preoperative and intraoperative components. We have evaluated the protocol as an entire system. It would be beneficial to investigate the effects of the individual protocol components separately to compare and separate, for example, the effects of the patient-selection process versus the effects of the refined intraoperative methods. The study we conducted was an initial step and assisted in answering our initial research questions. More granular and detailed research that can address the specific effects of individual protocol components is a possibility for future work.

TABLE 3. Risk of any complication 30 days and 1 year after complex spine surgery, according to study period

\begin{tabular}{cccc}
\hline & \multicolumn{2}{c}{ No. of Patients (\%) } & \\
\cline { 2 - 3 } $\begin{array}{c}\text { Any Overall } \\
\text { Complication }\end{array}$ & $\begin{array}{c}\text { Preintervention } \\
\text { Period }\end{array}$ & $\begin{array}{c}\text { Postintervention } \\
\text { Period }\end{array}$ & $\begin{array}{c}\text { RR } \\
(95 \% \mathrm{Cl})^{*}\end{array}$ \\
\hline 30 days & $34(48)$ & $17(25)$ & $0.49(0.30-0.78)$ \\
\hline $1 \mathrm{yr}$ & $19(27)$ & $16(23)$ & $0.98(0.55-1.77)$ \\
\hline
\end{tabular}

* Analyses were adjusted for age ( $<65$ or $\geq 65$ years) and Charlson/Deyo comorbidity score ( 0 or $\geq 1)$.

\section{Implications for Practice}

System-based improvements in complex spinal surgery reduce the short-term postoperative risk of complications and death. We suggest that complex spine surgery practitioners and administrators consider designing and implementing systematic approaches to improve the safety of the services they offer. Our experience might be transferrable to the management of perioperative processes in other complex surgical fields and might help contribute to further reductions in complication rates within these other specialties.

As hospitals and practitioners are faced with increasing pressure to maximize quality and as patients are motivated to seek the best care, implementing structured systematic multidisciplinary approaches to delivering surgical care might become a safer standard. We encourage other institutions and practitioners to develop their own comprehensive approaches to reviewing the appropriateness and risk of surgical care for their patients. These types of systematic approaches to improving quality and mitigating risk might set institutions apart as health care quality leaders.

\section{Directions for Future Research}

The results of this evaluation have revealed a number of possible avenues for future research. Future studies might increase statistical power and address additional issues of confounding by leveraging larger surgical registries or by combining data from multiple institutions. It might also be useful to conduct similar investigations in larger populations to better differentiate longer-term differences in complication rates or those in populations within other institutions or health systems to determine reproducibility and generalizability for attaining meaningful improvements in both short- and long-term treatment outcomes. 


\section{Acknowledgments}

Dr. Wernli and Ms. Anderson had full access to all of the data in the study and take responsibility for the integrity of the data and the accuracy of the data analysis.

This research was supported by the Group Health Partnership for Innovation and Group Health Research Institute Development Fund awards.

\section{References}

1. Ames CP, Barry JJ, Keshavarzi S, Dede O, Weber MH, Deviren V: Perioperative outcomes and complications of pedicle subtraction osteotomy in cases with single versus two attending surgeons. Spine Deform 1:51-58, 2013

2. Baldus CR, Bridwell KH, Lenke LG, Okubadejo GO: Can we safely reduce blood loss during lumbar pedicle subtraction osteotomy procedures using tranexamic acid or aprotinin? A comparative study with controls. Spine (Phila Pa 1976) 35:235-239, 2010

3. Beebe M: CPT 2009: Current Procedural Terminology. Chicago: American Medical Association, 2008

4. Bertram W, Harding I: Complications of spinal deformity and spinal stenosis surgery in adults greater than 50 years old. J Bone Joint Surg Br 94:105, 2012

5. Buchlak QD, Yanamadala V, Leveque JC, Sethi R: Complication avoidance with pre-operative screening: insights from the Seattle spine team. Curr Rev Musculoskelet Med 9:316-326, 2016

6. Buck CJ: ICD-9-CM for Physicians. St. Louis: Elsevier Saunders, 2014

7. Charosky S, Guigui P, Blamoutier A, Roussouly P, Chopin $\mathrm{D}$ : Complications and risk factors of primary adult scoliosis surgery: a multicenter study of 306 patients. Spine (Phila Pa 1976) 37:693-700, 2012

8. Cho SK, Bridwell KH, Lenke LG, Yi JS, Pahys JM, Zebala LP, et al: Major complications in revision adult deformity surgery: risk factors and clinical outcomes with 2- to 7-year follow-up. Spine (Phila Pa 1976) 37:489-500, 2012

9. Cima R, Dankbar E, Lovely J, Pendlimari R, Aronhalt K, Nehring S, et al: Colorectal surgery surgical site infection reduction program: a national surgical quality improvement program-driven multidisciplinary single-institution experience. J Am Coll Surg 216:23-33, 2013

10. Deyo RA, Mirza SK, Martin BI, Kreuter W, Goodman DC, Jarvik JG: Trends, major medical complications, and charges associated with surgery for lumbar spinal stenosis in older adults. JAMA 303:1259-1265, 2010

11. Faber M, Bosch M, Wollersheim H, Leatherman S, Grol R: Public reporting in health care: how do consumers use quality-of-care information? A systematic review. Med Care 47:1-8, 2009

12. Fisher CG, Vaccaro AR, Mulpuri K, Angevine PD, Thomas KC, Patel AA, et al: Evidence-based recommendations for spine surgery. Spine (Phila Pa 1976) 37:E3-E9, 2012

13. Halpin RJ, Sugrue PA, Gould RW, Kallas PG, Schafer MF, Ondra SL, et al: Standardizing care for high-risk patients in spine surgery: the Northwestern high-risk spine protocol. Spine (Phila Pa 1976) 35:2232-2238, 2010

14. Howe CR, Agel J, Lee MJ, Bransford RJ, Wagner TA, Bellabarba $\mathrm{C}$, et al: The morbidity and mortality of fusions from the thoracic spine to the pelvis in the adult population. Spine (Phila Pa 1976) 36:1397-1401, 2011

15. Lee MJ, Hacquebord J, Varshney A, Cizik AM, Bransford
RJ, Bellabarba C, et al: Risk factors for medical complication after lumbar spine surgery: a multivariate analysis of 767 patients. Spine (Phila Pa 1976) 36:1801-1806, 2011

16. Porter ME: What is value in health care? N Engl J Med 363:2477-2481, 2010

17. Ross TR, Ng D, Brown JS, Pardee R, Hornbrook MC, Hart G, et al: The HMO Research Network Virtual Data Warehouse: a public data model to support collaboration. EGEMS (Wash DC) 2:1049, 2014

18. Sansur CA, Smith JS, Coe JD, Glassman SD, Berven SH, Polly DW Jr, et al: Scoliosis Research Society morbidity and mortality of adult scoliosis surgery. Spine (Phila Pa 1976) 36:E593-E597, 2011

19. Schwab FJ, Hawkinson N, Lafage V, Smith JS, Hart R, Mundis G, et al: Risk factors for major peri-operative complications in adult spinal deformity surgery: a multi-center review of 953 consecutive patients. Eur Spine J 21:2603-2610, 2012

20. Sethi RK, Pong RP, Leveque JC, Dean TC, Olivar SJ, Rupp SM: The Seattle Spine Team approach to adult deformity surgery: a systems-based approach to perioperative care and subsequent reduction in perioperative complication rates. Spine Deform 2:95-103, 2014

21. Street JT, Lenehan BJ, DiPaola CP, Boyd MD, Kwon BK, Paquette SJ, et al: Morbidity and mortality of major adult spinal surgery. A prospective cohort analysis of 942 consecutive patients. Spine J 12:22-34, 2012

22. Sugrue PA, Halpin RJ, Koski TR: Treatment algorithms and protocol practice in high-risk spine surgery. Neurosurg Clin N Am 24:219-230, 2013

23. Werner BC, Shen FH, Shimer AL: Infections after lumbar spine surgery: avoidance and treatment. Semin Spine Surg 23:142-150, 2011

24. Yadla S, Maltenfort MG, Ratliff JK, Harrop JS: Adult scoliosis surgery outcomes: a systematic review. Neurosurg Focus 28(3):E3, 2010

\section{Disclosures}

The authors report no conflict of interest concerning the materials or methods used in this study or the findings specified in this paper.

\section{Author Contributions}

Conception and design: Sethi, Buchlak, Anderson, Baldwin, Leveque, Shea, Ross, Wernli. Acquisition of data: Anderson, Baldwin, Shea, Ross. Analysis and interpretation of data: Sethi, Buchlak, Anderson, Baldwin, Leveque, Edwards, Shea, Ross, Wernli. Drafting the article: Sethi, Buchlak, Yanamadala, Anderson, Baldwin, Leveque, Shea, Ross, Wernli. Critically revising the article: Sethi, Buchlak, Yanamadala, Mecklenburg, Leveque, Edwards. Reviewed submitted version of manuscript: Sethi, Buchlak, Yanamadala, Anderson, Baldwin, Mecklenburg, Leveque, Edwards, Ross, Wernli. Statistical analysis: Anderson, Baldwin. Administrative/technical/material support: Sethi, Buchlak, Anderson, Edwards, Shea, Ross, Wernli. Study supervision: Sethi, Buchlak, Wernli.

\section{Correspondence}

Rajiv Sethi, Neuroscience Institute, Virginia Mason Medical Center, 1201 Terry Ave., Seattle, WA 98101. email: rajiv.sethi@ virginiamason.org. 